\title{
The youngest stellar clusters
}

\section{Clusters associated with massive protostellar candidates ${ }^{\star}$}

\author{
M. S. N. Kumar ${ }^{1}$, E. Keto ${ }^{2}$, and E. Clerkin ${ }^{1, \star \star}$ \\ 1 Centro de Astrofísica da Universidade do Porto, Rua das Estrelas, 7150-462 Porto, Portugal \\ e-mail: nanda@astro.up.pt \\ 2 Harvard Smithsonian Center for Astrophysics, 60 Garden Street, Cambridge, Massachusetts, USA
}

Received 21 March 2005 / Accepted 19 December 2005

\section{ABSTRACT}

We report on the identification of 54 embedded clusters around 217 massive protostellar candidates of which 34 clusters are new detections. The embedded clusters are identified as stellar surface density enhancements in the $2 \mu \mathrm{m}$ All Sky Survey (2MASS) data. Because the clusters are all associated with massive stars in their earliest evolutionary stage, the clusters should also be in an early stage of evolution. Thus the properties of these clusters should reflect properties associated with their formation rather than their evolution. For each cluster, we estimate the mass, the morphological type, the photometry and extinction. The clusters in our study, by their association with massive protostars and massive outflows, reinstate the notion that massive stars begin to form after the first generation of low mass stars have completed their accretion phase. Further, the observed high gas densities and accretion rates at the centers of these clusters is consistent with the hypothesis that high mass stars form by continuing accretion onto low mass stars.

Key words. stars: formation - HII regions - open clusters and associations: general

\section{Introduction}

Embedded stellar clusters, those clusters that are still surrounded by the molecular clouds in which they formed, are the youngest of the stellar clusters. As such, the embedded clusters are of particular interest to understand which properties of stellar clusters are related to their origins and which are derived from subsequent evolution (Elmegreen et al. 2000; Lada \& Lada 2003). For example, the mass segregation, the concentration of higher mass stars in the centers of clusters, that is observed in many optically visible open clusters is also seen in some of the embedded clusters. Because the embedded clusters are too young to have undergone significant dynamical evolution, the mass segregation must be a property of the process of star formation in the clusters. As another example, open clusters exhibit both hierarchical and centrally condensed morphological types. Observations of both morphological types in embedded clusters suggests that the morphology of the clusters reflects the morphology of the clouds from which the stars formed rather than the dynamical evolution of the cluster. Finally, the distribution of stellar masses in the embedded

\footnotetext{
* Full Fig. 1 is only available in electronic form at http://www. edpsciences.org

$\star \star$ Present address: Shanghai Institute of Applied Maths and Mechanics, Shanghai University, Shanghai 200072, PR China.
}

clusters ought to be little affected by evolution and therefore closest to the initial mass function (IMF).

While the embedded clusters are thought to be among the youngest clusters, if we were to identify a class of clusters in which star formation, and therefore the formation of the cluster itself, were just beginning, we would potentially be able to address some of the questions as to the causes and origins of some of the cluster properties. For example, observations of embedded clusters may suggest star formation rather than dynamical evolution as a cause of mass segregation, but there remains the question of the cause. Is mass segregation a result of the formation of massive stars by the collisions and coalescence of lower mass stars because collisions will be more common in the high stellar density in the center of a cluster (Bonnell et al. 1998; Testi et al. 1999)? Is mass segregation a result of the formation of massive stars by continuing accretion onto existing low mass stars (Beech \& Mitalas 1994; Behrend \& Maeder 2001; Bernasconi \& Maeder 1996; Meynet \& Maeder 2000; Keto 2003), a process that requires rapid accretion to overcome radiation and thermal pressure, and therefore requires dense gas as would be found in the center of a dense molecular cloud? Similarly, observations of actively forming clusters might potentially address the relationship of the star formation to molecular cloud structure. What is the difference in cloud structure leading to the hierarchical and centrally condensed morphological types of clusters? Do stars always form first in 
the center of a molecular cloud or in gravitationally collapsing fragments throughout the cloud? Finally, if the lower mass stars form first as suggested by several studies of open clusters (Herbig 1962; Stahler 1985), and required by the theories of massive star formation either by coalescence or continuing accretion, then observations of clusters in formation may potentially address the origins of the IMF. Observations of the stellar mass distribution in actively forming clusters may allow the opportunity to see mass distributions that are still evolving toward an IMF.

In this study, we test a hypothesis that a class of actively forming clusters, the youngest subset of the young embedded clusters, may be identified by searching for stellar clusters around previously identified massive protostellar candidates in isolated molecular clouds. These massive protostellar candidates, massive stars in their earliest stages of formation, have been identified as a class of luminous objects having specific IRAS colors that are associated with other indicators of massive star formation such as dense gas and dust, water masers, and ultra-compact HII (UCHII) regions (Palla et al. 1991; Molinari et al. 1996, 1998, 2000; Sridharan et al. 2001; Beuther et al. 2002a) and associated with isolated molecular clouds. These candidates are sources deeply embedded in their molecular clouds and therefore probably represent the first massive stars to form within the clouds. If there were more evolved massive stars in these clouds, then we would expect their winds, radiation pressure, and supernovae explosions to have at least partially cleared the region of gas and dust revealing perhaps a classic open cluster as would typically be found in a more evolved massive star forming region. Thus because the massive protostellar candidates are the first massive stars to form within their host molecular clouds, any associated clusters should represent clusters in their earliest evolutionary phase.

Our study is similar in its objectives to a previous survey of stellar clusters around Herbig Ae/Be stars (Testi et al. 1997, 1999). That study also sought to identify very young clusters with active star formation using the Herbig Ae/Be stars, which are protostars, as indicators of active star formation. In our study we use the massive protostellar candidates as indicators of the earliest stages of stellar and therefore cluster formation.

A number of independent near-infrared (NIR) observations of the regions around several of the massive protostellar candidates in our target list already show evidence for embedded stellar clusters (references in Table 2). In contrast to these previous observations, in this study, we undertake a systematic search for embedded clusters around all the previously identified high mass protostellar candidates in the lists of Molinari et al. (1996) and Sridharan et al. (2001).

We identify the potential clusters as star count density enhancements above the mean background level (Lada \& Lada 1995; Carpenter et al. 2000; Ivanov et al. 2002) using the existing $K$-band observations of the 2MASS database (Kleinmann et al. 1994). We report on the identification of 54 clusters by this technique of which 34 are new detections. We estimate some basic properties that can be derived from the $J, H, K$ data in 2MASS. Finally we discuss some implications of newer theories of high mass star formation for the formation and evolution of clusters.

\section{Method}

The 217 targets selected for our study represent the unique sources from the combined lists of 163 candidates in Molinari et al. (1996) and 69 in Sridharan et al. (2001). We searched for clusters within an area of $400^{\prime \prime} \times 400^{\prime \prime}$ around each of the 217 positions of high mass protostellar candidates.

The 2MASS database contains $J, H$, and $K$ bands, and we used the $K$-band at $2.2 \mu \mathrm{m}$ for the initial identification of the clusters because $K$-band suffers the least extinction. We constructed stellar surface density maps by spatially binning the 2MASS point sources. We selected sources with quality flags of $\mathrm{A}, \mathrm{B}, \mathrm{C}$, and D, excluding lower quality sources. We used bins of $120^{\prime \prime}$ separated by $60^{\prime \prime}$ to cover the $400^{\prime \prime} \times 400^{\prime \prime}$ area. Clusters were detected as star count density enhancements above the mean background level within the sampled area. We used the average noise $(\sigma)$ in the map as a reference and plotted contours of mode $+2 \sigma$ and above to identify clusters. The mode value represents the average star count in the region, and mode $+2 \sigma$ reveals the enhancement with respect to the average background. We identified 63 possible clusters for which we retrieved data over a larger area of $600^{\prime \prime} \times 600^{\prime \prime}$ in all the three photometric bands to enable better sampling of the background counts and also to construct color-color diagrams and color-magnitude diagrams. Based on improved sampling of the background that reduced the noise in the contour maps, and iterating with different bin sizes, we excluded some targets that had loose groupings of 5-8 stellar sources. After excluding such weak groupings, we identified 54 clusters all of which contain more than 8 stars, although the majority of the clusters show star counts in excess of 20-30. We then used a smaller bin-size of $80^{\prime \prime}$ (step size $40^{\prime \prime}$ ) to reveal relatively small scale structures within the detected clusters. The resulting contour maps for each of these 54 clusters are presented in Fig. 1. The contour levels begin at mode $+2 \sigma$ and increase in steps of $1 \sigma$.

In order to estimate the number of stars in each cluster we must account for the inclusion of background and foreground stars within the defined cluster boundary. To estimate the combined background and foreground stars, we sampled for each cluster an adjacent region of $1600^{\prime \prime 2}-2500^{\prime \prime 2}$. The corrected counts are listed in Table 2 as "true" cluster membership. If we assume that within the sensitivity of 2MASS data we might not detect any stars behind the high extinction of a massive star forming cluster, then our subtraction of the estimated count of background stars would result in an underestimate of the number of true cluster members. Thus our estimate of the numbers of stars in a cluster might represent a lower limit to the true number in cases where the extinction through the clusters is very high.

\subsection{Detection statistics}

We detected 54 clusters out of 217 unique sources which implies an overall $\sim 25 \%$ detection rate. However, there are no clusters detected in the RA range $6 \mathrm{~h}$ to $20 \mathrm{~h}$ that coincides with the Galactic mid-plane although some targets were associated with $K$-band nebulosity. Our technique of identifying clusters as stellar density enhancements in the 2MASS data 

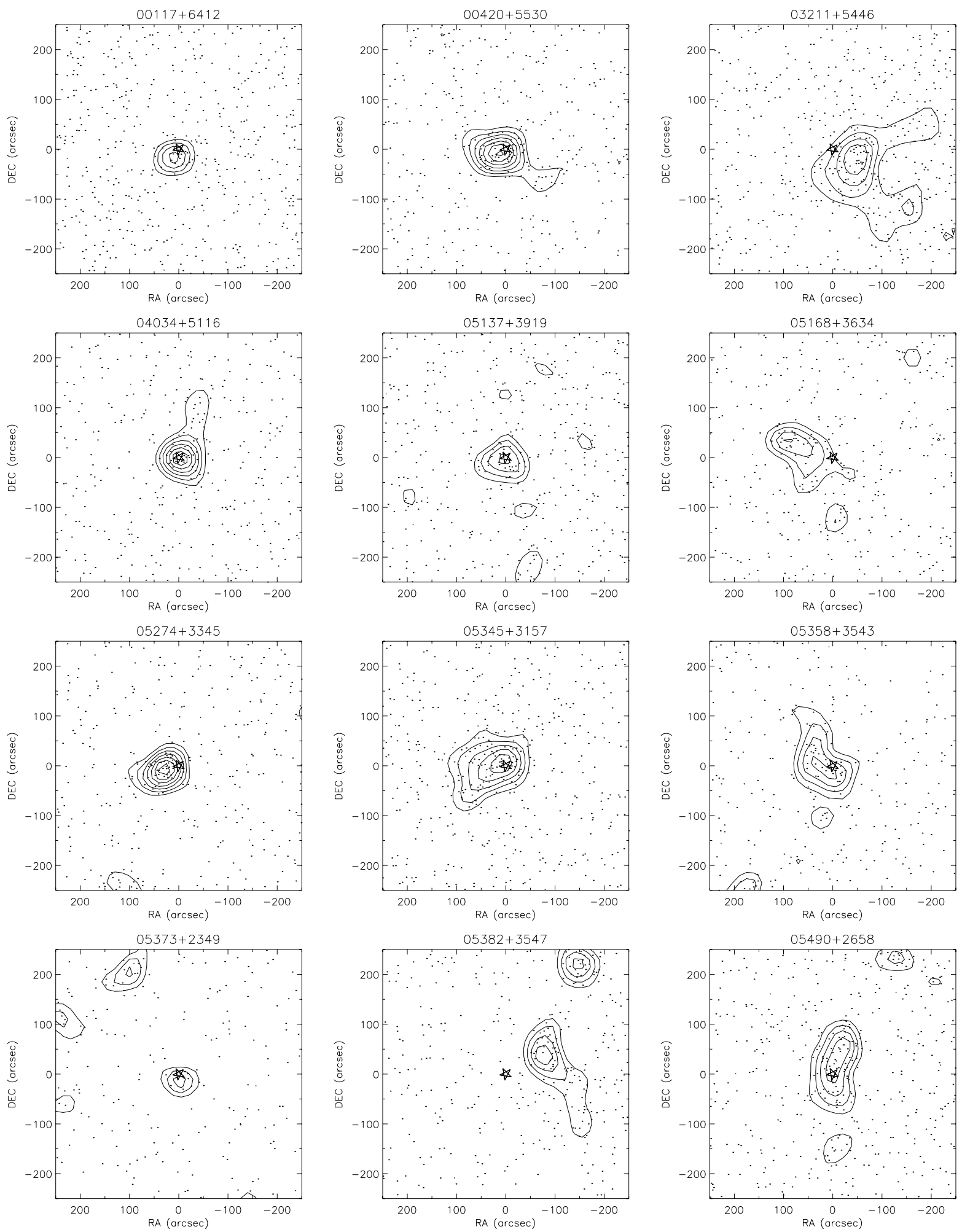

Fig. 1. Stellar surface density contour plots for the 54 embedded clusters produced with a bin size of $80^{\prime \prime}$ (step size $=40^{\prime \prime}$ ). The star symbol represents the position of the luminous IRAS source. Contours begin at mode $+2 \sigma$ level and increase in steps of $1 \sigma, \sigma$ being the average noise measured over the an area of $600^{\prime \prime} \times 600^{\prime \prime}$ centered on each target. The dots represent the point sources with phqual = A, B, C, D catalogued in the 2MASS GATOR database. The full figure is available at http://www. edpsciences.org/.

does not appear suitable to detect clusters in the Galactic midplane. First, the high extinction in the Galactic mid-plane reduces the number of stars that we can detect. Second, in the Galactic mid-plane the background count level is high and star count density enhancements are less significant with respect to the background. Excluding the targets in the 6-20 h RA range, the detection rate for clusters around the massive protostellar candidates is $60 \%$. 


\subsection{Cluster membership and properties}

In Table 1 we list the radius, distance, and luminosity of each cluster. The radius is defined from the area enclosed by the mode $+2 \sigma$ contours as $R=\sqrt{\text { area }} / \pi$. The distance and luminosity are generally quoted from their source papers namely MBCP96 and Sri01. Where available, improved distance estimates are used based on a SIMBAD search. In particular, targets beyond the Solar circle are relatively less studied making their distance estimates limited to the values quoted by Wouterloot et al. (1993). A histogram of the effective radii of the clusters indicates a mean cluster radius of $\sim 1 \mathrm{pc}$. The IRAS peak positions and the cluster peak positions coincide in about half of the detected sample and do not coincide in the remaining half. However, the fraction in which the two peaks coincide, the clusters are noted to be densest and are more circularly defined than the rest.

\subsection{Cluster mass estimation}

We estimated the mass of each cluster based on the method described by Lada \& Lada (2003, Hereafter LL03). The method incorporates the $K$-luminosity function (KLF) models of Muench et al. (2002) and the evolutionary tracks of D' Antona \& Mazitelli (1994). The mass estimation is done by posing the following question. If we were to place the Trapezium cluster at the same distance as one of the sample clusters and subject it to the same dust extinction, how many of its 780 members (see Muench et al. 2002) are we likely to observe? This question can be trivially answered if we know the completeness limit (limiting magnitude) of our observed data and by using D' Antona \& Mazitelli (1994) tracks along with the formula

$M_{k}=K_{\mathrm{mag}}-5+5 \log ($ distance $)-A_{k}$

We then compute a ratio of the observed number of stars to the expected number if our cluster were the Trapezium personified. This ratio then merely indicates the ratio of the mass of the sample cluster to that of the mass of the Trapezium cluster which is assumed to be $413 M_{\odot}$ (Muench et al. 2002).

We use the individual extinction values to each cluster as derived from the color-color diagrams (see Sect. 2.5) of each cluster. Further, the 2MASS limiting magnitude in $K$-band of $15 \mathrm{mag}$ and an average cluster age of $1 \mathrm{Myr}$ is adopted to compute the masses. The resulting mass estimates are listed in Table 1. Figure 2 shows the embedded cluster mass distribution function (ECMDF) constructed using the cluster mass estimates in Table 1. The solid line represents the ECMDF for the sample from our work and the dotted line shows the LL03 ECMDF for their sample of embedded clusters within $2 \mathrm{kpc}$ from the Sun. The ECMDF of both samples are similar.

In Fig. 3 we plot the IRAS luminosity of the targets versus the mass of the associated embedded cluster. There is a correlation between the mass of the associated embedded cluster and the luminosity of the target massive protostar, similar to the correlation found in HAeBe stars by Testi et al. (1999).

\subsection{The morphology of embedded clusters}

In their review of embedded clusters, Lada \& Lada (2003) suggest that the embedded clusters can be classified into two morphological types, hierarchical and centrally condensed, and they suggest that these structures may reflect the physical processes responsible for cluster formation. In particular, the two types may indicate a dominance of turbulent (hierarchical) or gravitational (centrally condensed) energies. The embedded clusters, and particularly the youngest clusters still in formation, offer the opportunity to test this hypothesis against observations. Such a test would best be done comparing the structure of the stellar distribution of the clusters with the gas distribution in the clouds. This comparison would require observations outside the scope of this current research. However, we may compare the relative numbers of hierarchical and centrally condensed clusters in the two samples of embedded clusters, those identified in our current research as the youngest by their association with the massive protostellar candidates and those embedded clusters within $2 \mathrm{kpc}$ of the Sun, from the list in Lada \& Lada (2003). We classify the clusters that show single peaks in the stellar density maps as centrally symmetric (C-type) and those which show more than one peak as hierarchically structured (H-type). The results for our sample of clusters (Fig. 1) indicate a ratio H-type/C-type $\sim 0.8$. We applied the same classification scheme to the 70 embedded clusters of Lada \& Lada (2003), using stellar density maps produced from the 2MASS data by our procedures as described in Sect. 2 . The nearby embedded clusters have a ratio H-type/C-type $\sim 0.9$, essentially the same as their younger counterparts.

\subsection{Photometric analysis}

Once the clusters were detected, we constructed $H-K$ vs. $J-H$ color-color and $H-K$ vs. $K$ color-magnitude (CM) diagrams for each of the clusters to investigate the nature of the associated point sources. To do this, we first transformed the 2MASS data into the Bessel \& Brett system using the transformations:

$$
\begin{aligned}
& (H-K)_{\mathrm{BB}}=1.0298 \times(H-K)_{2 \mathrm{MASS}}-0.0350 \\
& (J-H)_{\mathrm{BB}}=1.0101 \times(J-H)_{2 \mathrm{MASS}}-0.0495 \text { and } \\
& K_{\mathrm{BB}}=1.0298 \times K_{2 \mathrm{MASS}}-0.0350 .
\end{aligned}
$$

We plotted all points that fell within the mode $+1 \sigma$ contour to allow consideration of even those members that fell at the extreme edges of the clusters along with the main-sequence dwarf and giant tracks, reddening vectors, T-Tauri locus (Meyer et al. 1997), and the HAeBe locus (Lada \& Adams 1992). We dereddened the points that fell within the reddening vectors enclosed by the main-sequence tracks to the M6-K6 locus in order to find the extinction to each point. A histogram of all such extinction values and an average extinction was constructed for each cluster. These values are listed in Table 2 and are used to obtain the corresponding mass estimates for each cluster. We also constructed $H-K$ vs. $K$ color-magnitude diagrams for each cluster and verified the consistency of extinction derived from color-color diagrams. 
Table 1. Properties of embedded clusters associated with candidate HMPOs.

\begin{tabular}{|c|c|c|c|c|c|c|c|c|c|c|}
\hline \multirow{2}{*}{$\begin{array}{l}\mathrm{EC}^{a} \\
\text { No. }\end{array}$} & \multirow{2}{*}{$\begin{array}{l}\text { IRAS } \\
\text { name }\end{array}$} & \multirow{2}{*}{$\begin{array}{r}d \\
\mathrm{kpc}\end{array}$} & \multirow{2}{*}{$\begin{array}{r}L \\
\log L_{\odot} \\
\end{array}$} & \multicolumn{2}{|c|}{ Cluster members } & \multicolumn{2}{|c|}{ Effec radius } & \multirow{2}{*}{$\begin{array}{r}A_{k} \\
\text { mag }\end{array}$} & \multirow{2}{*}{$\begin{array}{r}M_{\text {stellar }} \\
M_{\odot}\end{array}$} & \multirow[t]{2}{*}{ Outflow ${ }^{b}$} \\
\hline & & & & $\operatorname{mode}+2 \sigma$ & true & & $\mathrm{pc}$ & & & \\
\hline$\overline{1 *}$ & $00117+6412$ & 1.8 & 3.1 & 26 & 13 & 66 & 0.57 & 0.3 & 18 & $\bar{Y}$ \\
\hline $2 *$ & $00420+5530$ & 4.3 & 4.1 & 71 & 38 & 96 & 3.6 & 0.6 & 380 & $\mathrm{Y}$ \\
\hline $3 *$ & $03211+5446$ & 4.54 & 4.5 & 131 & 41 & 101 & 2.21 & 0.6 & 471 & - \\
\hline $4^{*}$ & $04034+5116$ & 3.98 & 4.2 & 50 & 16 & 112 & 2.16 & 0.3 & 100 & - \\
\hline 5 & $05137+3919$ & 11.5 & 4.6 & 33 & 7 & 116 & 6.08 & 0.3 & 1132 & $\mathrm{Y}$ \\
\hline $6^{*}$ & $05168+3634$ & 6.08 & 4.4 & 48 & 18 & 115 & 3.37 & 0.4 & 355 & $\mathrm{Y}$ \\
\hline 7 & $05274+3345$ & 1.55 & 3.6 & 48 & 29 & 103 & 0.77 & 0.5 & 38 & Y \\
\hline 8 & $05345+3157$ & 2.6 & 3.6 & 95 & 36 & 126 & 1.09 & 0.6 & 122 & $\mathrm{Y}$ \\
\hline 9 & $05358+3543$ & 1.8 & 3.8 & 53 & 30 & 130 & 1.13 & 0.5 & 48 & Y \\
\hline $10 *$ & $05373+2349$ & 2.4 & 3.3 & 11 & 7 & 65 & 0.88 & 0.5 & 18 & Y \\
\hline $11^{*}$ & $05382+3547$ & 16.5 & 5.7 & 98 & 60 & 152 & 18.76 & 0.8 & - & - \\
\hline 12 & $05490+2658$ & 2.1 & 3.5 & 95 & 43 & 122 & 1.23 & 0.6 & 94 & $\mathrm{Y}$ \\
\hline 13 & $05553+1631$ & 2.5 & 3.8 & 80 & 22 & 104 & 1.24 & 0.4 & 56 & $\mathrm{Y}$ \\
\hline 14 & $06056+2131$ & 1.5 & 3.8 & 132 & 74 & 132 & 0.95 & 0.8 & 111 & Y \\
\hline 15 & $06061+2151$ & 2.0 & 1.4 & 105 & 49 & 145 & 0.07 & 1.0 & 144 & - \\
\hline $16^{*}$ & $06063+2040$ & 4.52 & 4.9 & 202 & 114 & 121 & 2.64 & 0.7 & 1474 & - \\
\hline 17 & $06068+2030$ & 1.5 & 4.7 & 68 & 34 & 96 & 0.69 & 0.6 & 45 & - \\
\hline 18 & $06103+1523$ & 4.3 & 4.63 & 50 & 24 & 118 & 2.64 & 0.3 & 211 & - \\
\hline 19 & $06104+1524$ & 4.7 & 4.68 & 50 & 24 & 124 & 2.81 & 0.3 & 217 & - \\
\hline $20 *$ & $06105+1756$ & 4.5 & 3.38 & 70 & 35 & 114 & 1.87 & 1. & 314 & - \\
\hline 21 & $06155+2319$ & 1.6 & 1.3 & 127 & 71 & 132 & 1.02 & 0.6 & 102 & - \\
\hline 22 & $06291+0421$ & 1.96 & 1.96 & 38 & 20 & 113 & 1.07 & 0.5 & 36 & - \\
\hline 23 & $06308+0402$ & 2.02 & 3.9 & 47 & 17 & 96 & 0.94 & 0.6 & 34 & $\mathrm{Y}$ \\
\hline $24 *$ & $06382+0939$ & 0.76 & 2.2 & 72 & 38 & 125 & 0.46 & 0.5 & 28 & - \\
\hline 25 & 06584-0852 & 4.48 & 3.9 & 32 & 16 & 108 & 2.33 & 0.4 & 145 & $\mathrm{Y}$ \\
\hline 26 & $20028+2903$ & 1.55 & 3.7 & 37 & 10 & 72 & 1.29 & 0.7 & 15 & - \\
\hline 27 & $20050+2720$ & 0.73 & 2.6 & 57 & 28 & 105 & 0.37 & 0.7 & 21 & $\mathrm{Y}$ \\
\hline $28 *$ & $20081+2720$ & 0.7 & 2.5 & 36 & 12 & 78 & 0.26 & 1.0 & 9 & - \\
\hline $29^{*}$ & $20205+3948$ & 4.5 & 4.5 & 44 & 17 & 74 & 1.61 & 0.6 & 190 & - \\
\hline 30 & $20286+4105$ & 3.72 & 3.6 & 44 & 7 & 58 & 1.00 & 0.8 & 63 & $\mathrm{Y}$ \\
\hline $31 *$ & $20319+3958$ & 1.6 & 3.8 & 32 & 9 & 68 & 0.53 & 1. & 17 & - \\
\hline $32 *$ & $20406+4555$ & 11.9 & 5.1 & 64 & 5 & 28 & 0.69 & 0.5 & 1766 & - \\
\hline $33 *$ & $20444+4629$ & 2.42 & 3.5 & 81 & 17 & 57 & 0.96 & 0.3 & 37 & $\mathrm{~N}$ \\
\hline $34 *$ & $21046+5110$ & 0.59 & 2.4 & 62 & 24 & 85 & 0.99 & 0.6 & 17 & - \\
\hline $35^{*}$ & $21078+5211$ & 1.49 & 4.1 & 113 & 50 & 113 & 0.81 & 0.6 & 66 & $\mathrm{Y}$ \\
\hline $36^{*}$ & $21202+5157$ & 6.78 & 4.8 & 44 & 3 & 84 & 2.77 & 0.5 & 98 & - \\
\hline $37 *$ & $21526+5728$ & 8.11 & 4.4 & 24 & 4 & 53 & 1.12 & 0.4 & 202 & - \\
\hline $38^{*}$ & $21548+5747$ & 7.1 & 4.4 & 15 & 5 & 38 & 1.3 & 0.3 & 140 & - \\
\hline 39 & $22134+5834$ & 2.6 & 4.1 & 52 & 26 & 104 & 1.31 & 0.4 & 72 & $\mathrm{Y}$ \\
\hline $40 *$ & $22147+5948$ & 7.29 & 4.4 & 59 & 5 & 106 & 2.26 & 0.4 & 178 & - \\
\hline $41^{*}$ & $22172+5549$ & 2.87 & 3.7 & 171 & 35 & 126 & 1.74 & 0.3 & 109 & Y \\
\hline $42 *$ & $22272+6358$ & 1.23 & 3.3 & 23 & 11 & 74 & 0.44 & 0.4 & 11 & - \\
\hline $43^{*}$ & $22305+5803$ & 5.4 & 4.1 & 36 & 3 & 83 & 2.16 & 0.4 & 43 & Y \\
\hline $44 *$ & $22506+5944$ & 5.7 & 4.3 & 38 & 15 & 91 & 2.52 & 0.5 & 280 & $\mathrm{Y}$ \\
\hline $45^{*}$ & $22551+6221$ & 0.7 & 3.2 & 229 & 129 & 152 & 0.51 & 0.8 & 98 & - \\
\hline $46^{*}$ & $22570+5912$ & 5.1 & 4.7 & 96 & 25 & 82 & 2.01 & 0.3 & 270 & $\mathrm{Y}$ \\
\hline $47 *$ & $23026+5948$ & 5.76 & 4.2 & 59 & 22 & 86 & 2.39 & 0.3 & 331 & $\mathrm{~N}$ \\
\hline 48 & $23033+5951$ & 3.5 & 4. & 10 & 3 & 74 & 1.26 & 0.3 & 14 & $\mathrm{Y}$ \\
\hline $49^{*}$ & $23146+5954$ & 4.43 & 4.4 & 56 & 19 & 119 & 2.55 & 0.3 & 153 & - \\
\hline $50 *$ & $23151+5912$ & 5.7 & 5. & 27 & 2 & 97 & 2.69 & 0.4 & 33 & Y \\
\hline $51 *$ & $23314+6033$ & 2.78 & 4. & 176 & 61 & 126 & 1.69 & 0.6 & 239 & $\mathrm{Y}$ \\
\hline $52 *$ & $23448+6010$ & 2.02 & 3.4 & 64 & 38 & 89 & 0.86 & 0.5 & 66 & - \\
\hline $53^{*}$ & $23507+6230$ & 4.28 & 4.1 & 23 & 8 & 88 & 1.81 & 0.4 & 66 & - \\
\hline $54 *$ & $23545+6508$ & 0.8 & 3.6 & 92 & 39 & 145 & 0.56 & 0.7 & 31 & - \\
\hline
\end{tabular}

$a *$ Denotes newly detected embedded clusters. The remaining clusters were known previously as listed below (5) Ishii et al. (2002); (7, 12, 17, 21, 23) Carpenter et al. (1993); (8) Chen et al. (1999); (18, 26, 30) Dutra \& Bica (2001); (9) Porras et al. (2000); (13, 14, 15) Carpenter et al. (1995); (22) Phelps \& Lada (1997); (25) Ivanov et al. (2002); (39, 48) Kumar et al. (2002).

${ }^{b}$ Outflow identifications from Beuther et al. (2002b), Zhang et al. (2005) and Wu et al. (2004). 


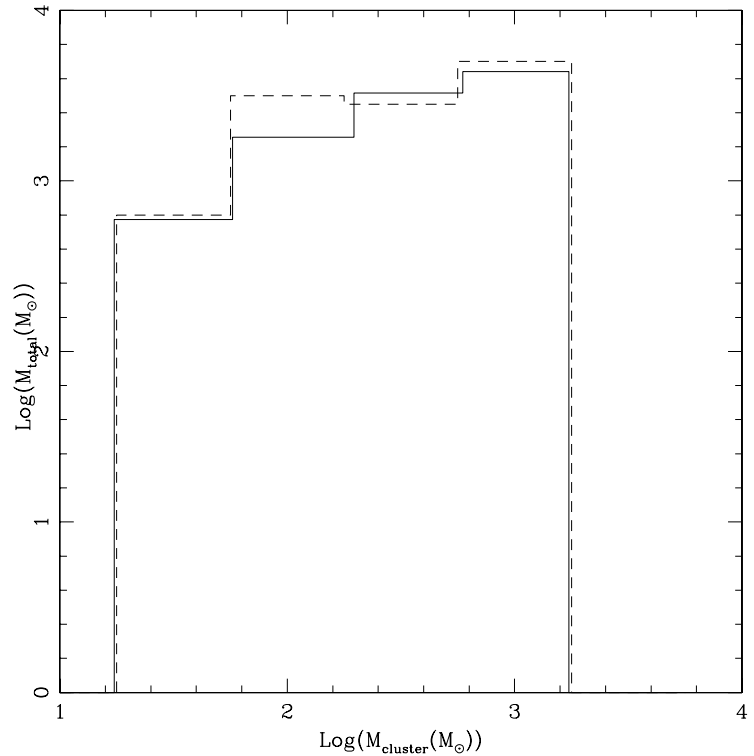

Fig. 2. Embedded cluster mass distribution function. The dotted line represent the ECMDF from LL03 for the embedded clusters within $2 \mathrm{kpc}$ distance. The solid line shows the ECMDF for the clusters from this work.

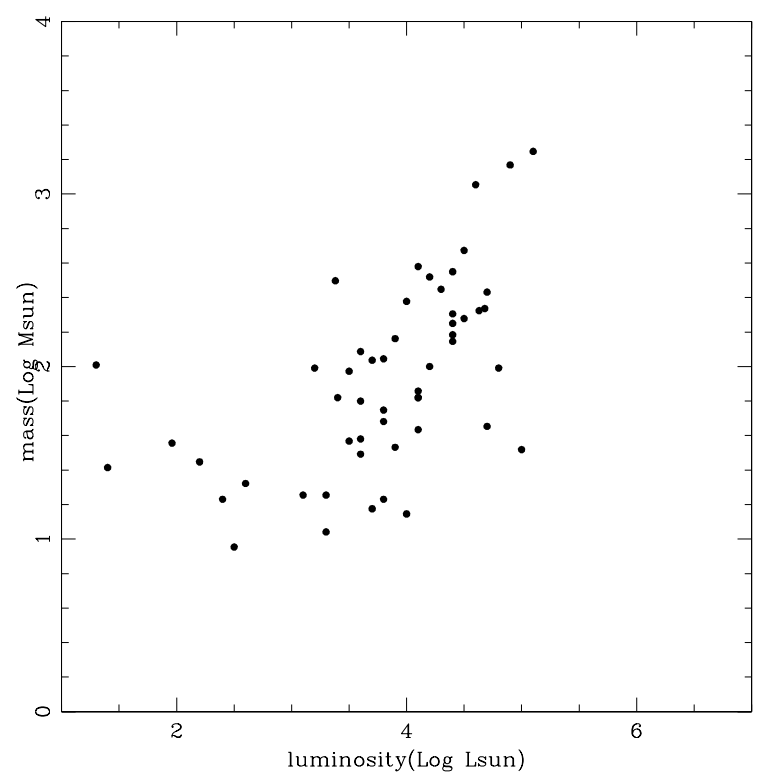

Fig. 3. A plot of the mass of the associated clusters vs. the luminosity of the target massive protostar.

\subsection{The NIR colors of massive protostars}

The massive protostellar candidates were all initially identified by their IRAS colors, and the list further refined by the association of the candidates with other signs of star formation (references in Introduction). We may ask whether the NIR colors of any of the clusters or their members are distinct.

For example, in the formation of high mass stars, it has been hypothesized that the dense accretion flows that form the stars suppresses the formation and later the expansion of an HII region (Walmsley 1995; Keto 2003). Once the HII region reaches a size detectable by typical VLA observations, the accretion flow will have ended and the star should have

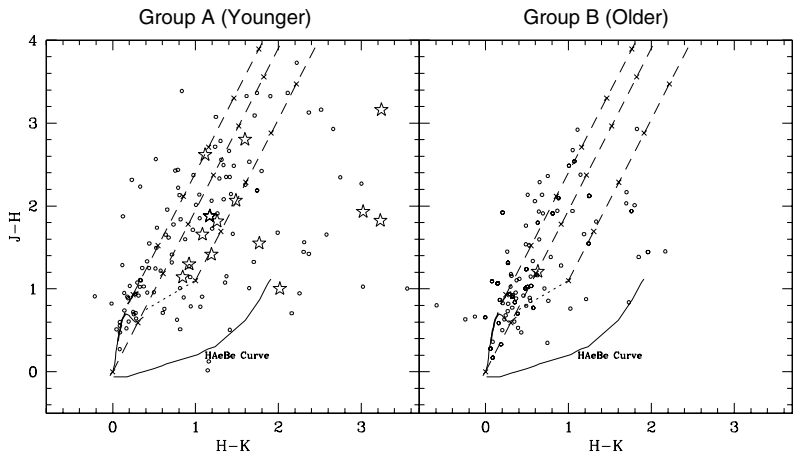

Fig. 4. Color-color diagram of NIR counterparts to the $1.2 \mathrm{~mm}$ peaks of HMPOs. The solid curve represents the HAeBe curve by Lada \& Adams (1992), thick-solid curve represents the main-sequence tracks, the dotted line shows the T-Tauri locus from Meyer et al. (1997) and the dashed lines represent the reddening vectors. The dots represent the point sources from GATOR PSC and star symbols represent the extended sources from the GATOR XSC.

obtained its main sequence mass. The list of massive protostellar candidates contains candidates associated with radio continuum emission (HII regions) and those without detectable radio continuum. In order to identify any differences between the NIR emission of these two types of sources, we searched for NIR emission within 5" of the dust continuum peaks mapped by Molinari et al. (2000) and Beuther et al. (2002) using both the 2MASS Point Source Catalog (PSC) and Extended Source Catalog (XSC). The extended NIR sources are shown as star symbols, and NIR point sources are shown as points in Fig. 4 plotted along with the main sequence dwarf \& giant loci, the T-Tauri locus and the HAeBe locus. Reddening vectors assume an interstellar reddening law of $R=3.12$. The sources with no radio continuum (Group A in Fig. 4) display very red colors, are spread around the region of the main sequence and T-Tauri loci, and extend way beyond the limits of the HAeBe locus. In comparison, the older sources are limited to the main-sequence region with some falling within the HAeBe locus. As far as this test goes, the data are consistent with the theory that the massive protostellar candidates without associated radio continuum are more deeply embedded than those with HII regions. The test is limited. The NIR emission, particularly the extended emission, is not necessarily associated with any massive protostellar candidate. The NIR emission is from regions of very high extinction that may affect the $J, H$, and $K$ magnitudes. Spectroscopic observations would be more reliable in identifying individual sources. However, the $J, H$, and $K$ data available in the 2MASS survey in combination with the continuum emission from dust may be sufficient to suggest the locations of the massive protostellar candidates without suggesting a correspondence with individual stars.

\section{Discussion}

\subsection{Implications for the relative ages of low and high mass stars}

If one accepts the hypothesis that the high mass protostellar candidates identified by the IRAS satellite can be localized by 
the observed combination of the dust continuum and NIR, then there is evidence for mass segregation in our sample of the youngest clusters still in formation. Furthermore if we accept the hypothesis that the massive protostellar candidates are the first massive stars to form in a cluster, the presence of the cluster itself, which is identified primarily through its more numerous lower mass members, suggests that the low mass stars form before their high mass counterparts. This hypothesis has been in the literature for quite some time (e.g. Herbig 1962). The clusters in our study, by their association with massive protostars, reiterate the suggestion with slightly different reasoning than presented earlier (e.g. Hillenbrand et al. 1993; Testi et al. 1999). The difference is that, in our sample the sources are relatively more massive than typical HAeBe stars, $\sim 40 \%$ of the detected clusters are also associated with massive outflows (see Table 2), infall signatures (Fuller et al. 2005) and many sources lack any significant HII regions. Therefore, the massive stars are still in accreting phases as evidenced by their outflows while the low mass members of the associated clusters have finished accreting and emerged into the Class I or II phases as evidenced by their $2 \mu \mathrm{m}$ appearance. Therefore our result places a stronger constraint that the massive stars begin to form at least after a few $10^{4} \mathrm{yr}$ after the first low mass stars in these embedded clusters were born.

\subsection{Implications for the formation mechanism of massive stars}

The previous study of stellar clusters associated with Herbig Ae/Be stars by Testi et al. (1997, 1999) suggested that the stellar spectral type of the Herbig Ae/Be star may be correlated with the richness (stellar density) of the cluster. This leads to the hypothesis that dynamical interaction (collisions) between low mass stars may be required to form a high mass star (Testi et al. 1999). That hypothesis was motivated in part by the high stellar densities observed. Our study does not contradict this suggestion, but our results also suggest an alternative hypothesis.

In our sample, those clusters in which the IRAS/mm peaks and the cluster peaks coincide most closely are also the densest clusters both in terms of stellar density as well as molecular gas density. The very high gas densities in the centers of these clusters, inferred from observations of maser and thermal molecular line emission and radio continuum emission are consistent with the hypothesis that the high mass stars form by continuing accretion onto lower mass stars. The hypothesis of continuing accretion requires very massive accretion flows, perhaps up to $10^{-3} M_{\odot} \mathrm{yr}^{-1}$ to form the earliest type stars. (Keto 2003). These massive flows are more likely found in very dense gas that our study, in conjunction with previous observations, locates at the centers of the clusters where the most massive stars are forming.

There are three principle reasons why the the hypothesis of continuing accretion requires a very high rate of accretion to form an early type star. First, the rate of accretion must be high enough to keep a star from evolving off the main sequence before it has reached a high mass (Keto 2003). Second, the momentum of the accretion flow $\dot{M} v$ must be high enough to overcome the high radiation pressure of the stellar luminosity (Kahn 1974; Wolfire \& Cassinelli 1987; Keto \& Wood 2006). Third, the density of the gas around the star must be high enough to keep the HII region around the star small so that the accretion flow is not reversed by the thermal pressure of the ionized gas. Specifically the radius of ionization equilibrium must be within the distance where the escape velocity from the star equals the ionized sound speed (Keto 2002a,b).

The hypothesis of continuing accretion has specific implications for our understanding of stellar evolution and therefore cluster evolution. It is a fundamental assumption of the standard theory of stellar evolution that once the mass of a star is fixed, its subsequent evolution is determined. Stars that continue to gain mass by accretion obviously do not have a fixed mass, and their evolutionary tracks in color-magnitude space or the Hertzprung-Russell diagram are different than for nonaccreting stars, and furthermore depend on the rate of accretion. For example, the stellar structure calculations of Stahler et al. (1980a,b, 1981) showed that stars of mass greater than about $7 M_{\odot}$ begin hydrogen burning while still in the accretion phase and thus do not have a protostellar phase equivalent to that in lower mass stars. This result has been confirmed in subsequent calculations (Beech \& Mitalas 1994; Behrend \& Maeder 2001; Bernasconi \& Maeder 1996; Meynet \& Maeder 2000). Second, continuing accretion will supply fresh hydrogen to the star, and if the rate is high enough, the fresh hydrogen will prevent the star from evolving off the main sequence. Third for stars below about $7 M_{\odot}$, the accretion will provide fresh deuterium to the star and prolong the phase of deuterium shell burning, altering the pre-main sequence evolutionary tracks. We briefly discuss these differences in stellar evolution and their consequences for our understanding of cluster evolution.

The accretion rates and time scales necessary for an intermediate mass star to evolve to a high mass star can be estimated through stellar structure calculations. Figure 5 shows evolutionary tracks for stars with continuous accretion at various rates. The evolutionary tracks were calculated for us by Alessandro Chieffi based on the stellar structure models described in Chieffi et al. (1995). The evolutionary tracks are calculated for accretion rates normalized to $6 \times 10^{-8} M_{\odot} \mathrm{yr}^{-1}$ for a star of unity $M_{\odot}$ but with different dependencies on the stellar mass in each track. While the mode of accretion in high mass star formation is not well known, observations indicate that the rates may scale as a power of the stellar mass (Churchwell 1998; Henning 2000; Behrend \& Maeder 2001). We plot accretion rates scaling with powers of the stellar mass from 0.5 to 2.0. A rate proportional to the square of the mass is the maximum accretion rate attainable in purely spherical accretion (Bondi 1952).

The time scale for the formation of high mass stars is quite short. The time required for a star of $4.0 M_{\odot}$ to reach the point where it turns off the main sequence toward the giant branch is printed on the figure for each accretion rate. The main sequence lifetimes for stars of $\mathrm{B}$ to late $\mathrm{O}$ type are roughly between 0.5 and 1.0 Myr. The most massive $O$ stars spend such a short time in the accretion phase that observed examples of 


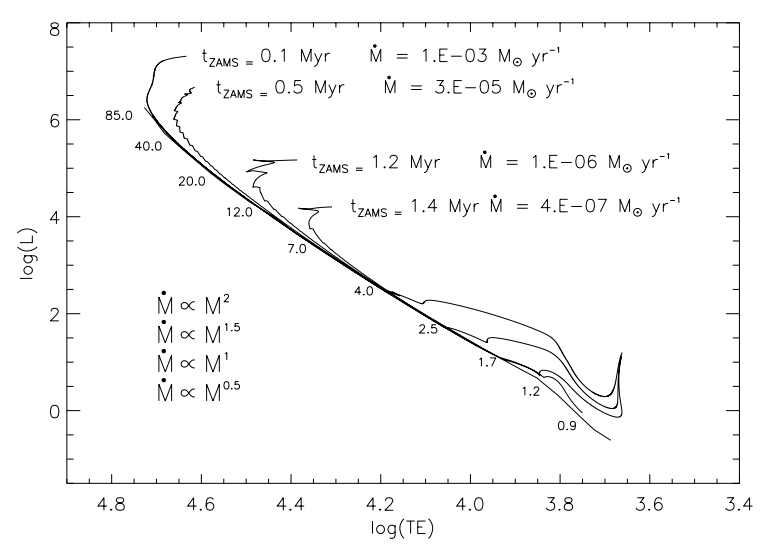

Fig. 5. An HR-diagram showing evolutionary tracks for massive stars that are gaining mass by accretion. The zero age main sequence (ZAMS) line is shown annotated by stellar mass in solar masses. The four evolutionary tracks are for stars accreting with rates $(\dot{M})$ that vary as a power law of stellar mass $(M)$, with indexes $0.5,1,1.5$ and 2 (bottom to top respectively). $t_{\mathrm{ZAMS}}$ indicates the time spent on the ZAMS line for a $4 M_{\odot}$ star before it deviates off the line. The accretion rate at the time of deviation, which is also the maximum accretion rate for that track is marked below each track.

early $\mathrm{O}$ stars in the accretion phase would be expected to be quite rare. Because the accretion rate scales as a power of the stellar mass, these precursors to massive stars spend most of their time in the lower mass range where the accretion rate is lower and the evolution proceeds more slowly. Thus we would not expect to see many $\mathrm{O}$ type stars in deeply embedded clusters that are at the earliest stages of formation.

Continuing accretion supplies fresh deuterium to the stars allowing deuterium burning in the outer shell of the star to continue past the time when the deuterium would have been exhausted in a non-accreting star. This keeps the stellar radius larger and thus the luminosity higher than for non-accreting stars. Thus pre-main sequence evolutionary tracks of accreting stars maintain a higher luminosity as the pre-main sequence star evolves (Fig. 5; see also Behrend \& Maeder 2001). At around $4 M_{\odot}$, the luminosities of both accreting and nonaccreting stars are no longer dominated by Deuterium shell burning and their luminosities are essentially the same. Thus as accreting stars above $4 M_{\odot}$ gain mass they appear to move up the main sequence as defined by the standard theory of nonaccreting stars. If all massive stars in a cluster form by continuing accretion onto lower mass stars, then there will be no massive pre-main sequence stars and no stars above $4 M_{\odot}$ in the HR diagram on high mass pre-main sequence tracks. HR diagrams from observations of clusters are consistent with this hypothesis (NGC 2264, NGC 6530 Stahler 1985; NGC 6611 Hillenbrand et al. 1993). In particular, these clusters show large numbers of stars with masses less than $4 M_{\odot}$ above the main sequence, but few stars above the main sequence at higher masses. These HR diagrams are of course also consistent with the stellar evolution of non-accreting stars. In particular, the absence of observed pre-main sequence massive stars could be due to the brevity of the pre-main sequence phase in massive stars. Nevertheless, the determination of the ages of stars and clusters by the location of the stars on the HR diagram already has some inherent uncertainty (Stahler 1985), and the possibility of continuing accretion adds further uncertainty to this method of age determination. Thus a method to date clusters by their association with protostars as in Testi et al. (1999) or by their association with massive protostellar candidates as in this study should be useful in distinguishing between the different hypotheses of accreting and non-accreting stellar evolution.

\subsection{Observational evidences for continuing accretion}

Finally, we note that several individual studies of massive protostars in recent years support the conjecture that massive stars are most likely formed by continuing accretion. First, continuing accretion requires that an intermediate mass star such as an A or B star forms by accretion and should be surrounded by an envelope mass much greater than the protostar itself, so that sufficient material is available to feed the intermediate mass star to build its mass. Second, the accretion rates should be high enough of the order of $10^{-3} M_{\odot} \mathrm{yr}^{-1}$. Detailed studies of sources such as IRAS20126+4104 (Cesaroni et al. 1994), IRAS20293+3952 (Beuther et al. 2004), IRAS07427-2400 (Kumar et al. 2003b), G31.41+0.31 and G24.78+0.08 (Beltran et al. 2004b) have shown the presence of both Keplerian disks and large toroids that contain several hundreds to a few thousand solar masses of material. In the case of IRAS07427-2400, the massive star is visible even at $2 \mu \mathrm{m}$ and is driving a powerful massive outflow. For instance, in the best studied example of IRAS20126+4104, while a mass of $7 M_{\odot}$ is estimated by using the observed Keplerain velocities, the luminosity derived from a spectral energy distribution curve $\left(\sim 10^{4} L_{\odot}\right)$ is an order of magnitude higher than that expected from a single $7 M_{\odot}$ star (Cesaroni et al. 2005). Further, the mass accretion rates derived from the above studies are in the range of $10^{-4} M_{\odot}$ to as high as $10^{-2} M_{\odot}$ (Beltran et al. 2004a, 2004b). The observational evidences consistently suggest a scenario where intermediate mass objects are surrounded by massive envelopes with sufficient accretion rates to form a massive star through continuing accretion. It should be noted that contrary to this scenario, in low mass star formation, the envelope mass is greater than the protostar mass only in Class 0 phase $\left(M_{\mathrm{env}}>M_{*}\right)$ and during Class I or II phases $M_{\mathrm{env}}<M_{*}$.

\section{Summary and conclusions}

We conducted a systematic search for clustering around 217 candidate HMPOs chosen from the combined lists of MBCP96 and Sri02. We used the 2MASS GATOR database and the technique of producing stellar surface density contours to detect clusters. We also searched for near-infrared counterparts of the $1.2 \mathrm{~mm}$ dust continuum peaks associated with all candidate HMPOs.

1. We find 54 embedded clusters associated with 217 candidate HMPOs indicating a $25 \%$ cluster detection rate. All targets lying in the Galactic mid-plane did not show any clusters, and we attribute this to the insensitivity of the 2MASS data to probe into the large extinctions in the Galactic mid-plane region. The detection rate for targets away from the mid-plane is $60 \%$. 
2. We estimate the mass of each cluster associated with massive protostellar candidates and find that the embedded cluster mass distribution function is similar to that found in a sample of all embedded clusters within $2 \mathrm{kpc}$ of the Sun.

3. Approximately equal numbers of clusters associated with massive protostellar candidates are found to have hierarchical as centrally condensed structures.

4. In about half of the detected sample, the cluster peaks and the IRAS/mm peaks coincide very well indicating a positive identification of the massive protostar with the infrared visible embedded clusters. This fraction of clusters also display the highest densities and more circular morphology in the entire sample.

5. One hypothesis of the formation of massive stars by continuing accretion is that the younger stars will not be associated with radio free-free emission from HII regions. We find that the near IR colors of sources near the dust continuum peaks and that do not show free-free emission are redder than those that are associated with ultra-compact HII regions.

6. The data are consistent with the hypothesis that massive stars form by continuing accretion, but the data do not discriminate against the hypothesis that massive stars form by the collisions of lower mass stars.

Acknowledgements. We are grateful to Alessandro Chieffi for computing the evolutionary tracks shown in Fig. 5. We also thank an anonymous referee for valuable suggestions that significantly improved the presentation of this paper. This publication makes use of data products from the Two Micron All Sky Survey, which is a joint project of the University of Massachusetts and the Infrared Processing and Analysis Center/California Institute of Technology, funded by the National Aeronautics and Space Administration and the National Science Foundation. This work was supported by grants POCTI/1999/FIS/34549 and POCTI/CFE-AST/55691/2004 approved by FCT and POCTI, with funds from the European Community programme FEDER.

\section{References}

Bachiller, R., \& Cernicharo, J. 1990, A\&A, 239, 276

Beech, M., \& Mitalas, R. 1994, ApJS, 95, 517

Behrend, A., \& Maeder, A. 2001, ApJS, 373, 190

Beltr'an, M. T., Cesaroni, R., Neri, R., et al. 2004a, ApJ, 601, L187

Beltr'an, M. T., Cesaroni, R., Neri, R., et al. 2004b, A\&A, 435, 901

Bernasconi, P., \& Maeder, A. 1996, A\&A, 307, 839

Beuther, H., Schilke, P., Sridharan, T. K., et al. 2002a, A\&A, 383, 892

Beuther, H., Schilke, P., Sridharan T. K., et al. 2002b, A\&A, 383, 892

Beuther, H., Schilke, P., \& Gueth, F. 2004, ApJ, 608, 330

Bondi, M. 1952, MNRAS, 112, 195

Bonnell, I. A., Bate, M., \& Zinnecker, H. 1998, MNRAS, 298, 93

Carpenter, J. M., Snell, R. L., Schloerb, F. P., \& Skrutskie, M. F. 1993, ApJ, 407, 657

Carpenter, J. M., Snell, R. L., \& Schloerb, F. P. 1995, ApJ, 450, 201

Carpenter, J. M. 2000, AJ, 120, 3139

Cesaroni, R., Olmi, L., Walmsley, C. M., Churchwell, E., \& Hofner, P. 1994, A\&A, 435, L137

Cesaroni, R., Neri, R., Olmi, L., et al. 2005, A\&A, 434, 1039

Chen, Y., Yao, Y., Yang, J., et al. 1999, AJ, 117, 446

Chieffi, A., Staniero, O., \& Salaris, M. 1995, ApJ, 445, L39
Churchwell, E. 1998, in The Origin of Stars and Planetary Systems, ed. C. Lada, \& N. Kylafis, NATO Science Series (Kluwer), 540, 515

D’ Antona, F., \& Mazitelli, I. 1994, ApJ, 90, 467

Dutra, C. M., \& Bica, E. 2001, A\&A, 376, 434

Elmegreen, B. G., Efremov, Y., Pudritz, R., \& Zinnecker, H. 2000, in Protostars \& Planets IV, ed. V. Mannings, A. P. Boss, \& S. S. Russell (University of Arizona Press), 179

Fuller, G. A., Williams, S. J., \& Sridharan, T. K. 2005, A\&A, 442, 949

Henning, T., Schreyer, K., Launhardt, R., \& Burkert, A. 2000, A\&A, 353,211

Herbig, G. H. 1962, ApJ, 135, 736

Hillenbrand, L. A., Massey, P., Strom, S. E., \& Merrill, K. M. 1993, AJ, 106, 1906

Hillenbrand, L. 1995, Ph.D. Thesis, University of Massachusetts

Jijina, J., \& Adams, F. 1996, ApJ, 462, 87

Ivanov, V. D., Borissova, J., Pessev, P., Ivanov, G. R., \& Kurtev, R. 2002, A\&A, 394, L1

Ishii, M., Hirao, T., Nagashima, C., et al. 2002, AJ, 124, 430

Kahn, F. 1974, A\&A, 37, 149

Keto, E. 2002a, ApJ, 568, 754

Keto, E. 2002b, ApJ, 580, 980

Keto, E. 2003, ApJ, 599, 1196

Keto, E., \& Wood, K. 2006, ApJ, 637, 850

Kleinmann, S. G., Lysaght, M. G., Pughe, W. L., et al. 1994, Exp. Astron., 3(1-4), 65

Kumar, M. S. N., Bachiller, R., \& Davis, C. J. 2002, ApJ, 576, 313

Kumar, M. S. N., Ojha, D. K., \& Davis, C. J. 2003, ApJ, 598, 1107

Kumar, M. S. N., Fernandes, A. J. L., Hunter, T. R., Davis, C. J., \& Kurtz, S. 2003, A\&A, 412, 175

Lada, C. J., \& Adams, F. C. 1992, ApJ, 393, 278

Lada, C. J., \& Lada, E. A. 2003, ARA\&A, 41, 57, LL03

Lada, E. A., \& Lada, C. J. 1995, AJ, 109, 1682

Meyer, M. R., Calvet, N., \& Hillenbrand, L. 1997, AJ, 114, 288

Meynet, G., \& Maeder, A. 2000, A\&A, 361, 101

Molinari, S., Brand, J., Cesaroni, R., \& Palla, F. 1996, A\&A, 308, 573 (MBCP96)

Molinari, S., Brand, J., Cesaroni, R., Palla, F., \& Palumbo, G. G. C. 1998, A\&A, 336, 339

Molinari, S., Brand, J., Cesaroni, R., \& Palla, F. 2000, A\&A, 355, 617

Muench, A. A., Alves, J. F., Lada, C. J., \& Lada, E. A. 2001, ApJ, 558, L51

Muench, A. A., Lada, E. A., Lada, C. J., \& Alves, J. F. 2002, ApJ, 573,366

Palla, F., Brand, J., Comoretto, G., Felli, M., \& Cesaroni, R. 1991, A\&A, 246, 249

Phelps, R. L., \& Lada, E. A. 1997, ApJ, 477, 176

Porras, A., Cruz-Gonzalez, I., \& Salas, L. 2000, A\&A, 361, 660

Sridharan, T. K., Beuther, H., Schilke, P., Menten, K. M., \& Wyrowski, F. 2001, ApJ, 566, 931, Sri01

Stahler, S., Shu, F., \& Taam, R. 1980a, ApJ, 241, 637

Stahler, S., Shu, F., \& Taam, R. 1980b, ApJ, 242, 226

Stahler, S., Shu, F., \& Taam, R. 1981, ApJ, 248, 727

Stahler, S. W. 1985, ApJ, 293, 207

Testi, L., Palla, F., Prusti, T., Natta, A., \& Maltagliti, S. 1997, A\&A, 320, 159

Testi, L., Palla, F., \& Natta, A. 1999, A\&A, 342, 515

Yorke, H., \& Sonnhalter, C. 2002, ApJ, 569, 846

Watson, A. M., Coil, A. L., Shepherd, D. S., Hofner, P., \& Churchwell, E. 1997, ApJ, 487, 818

Wolfire, M., \& Cassinelli 1987, ApJ, 319, 850

Wouterloot, J. G. A., Brand, J., \& Fiegle, K. 1993, A\&AS, 98, 589

Wu, Y., Wei, Y., Zhao, M., et al. 2004, A\&A, 426, 503

Zhang, Q., Hunter, T. R., Brand, J., et al. 2005, ApJ, 625, 864 


\section{Online Material}


M. S. N. Kumar et al.: The youngest stellar clusters, Online Material p 2
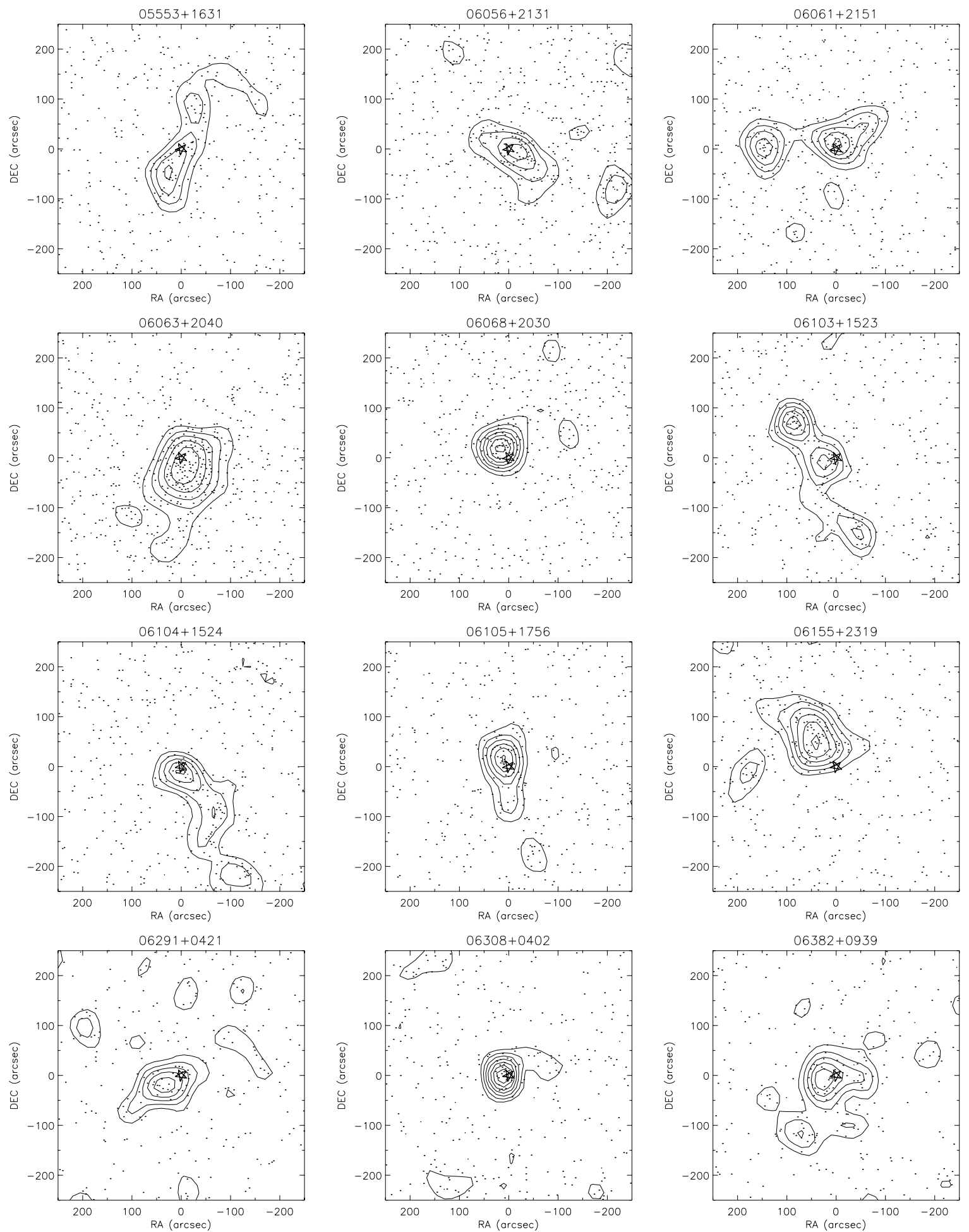

Fig. 1. continued. 
M. S. N. Kumar et al.: The youngest stellar clusters, Online Material p 3
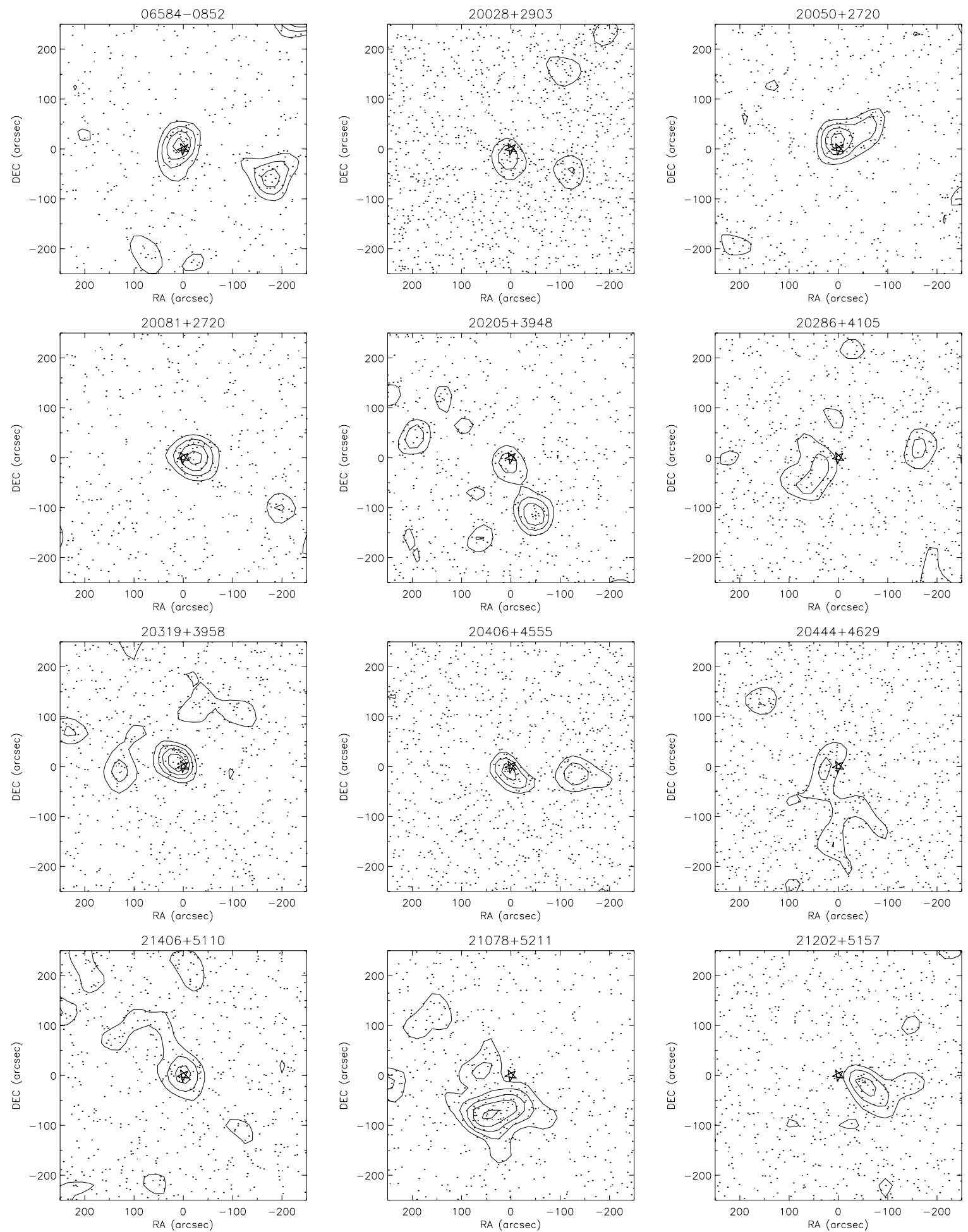

Fig. 1. continued. 
M. S. N. Kumar et al.: The youngest stellar clusters, Online Material p 4
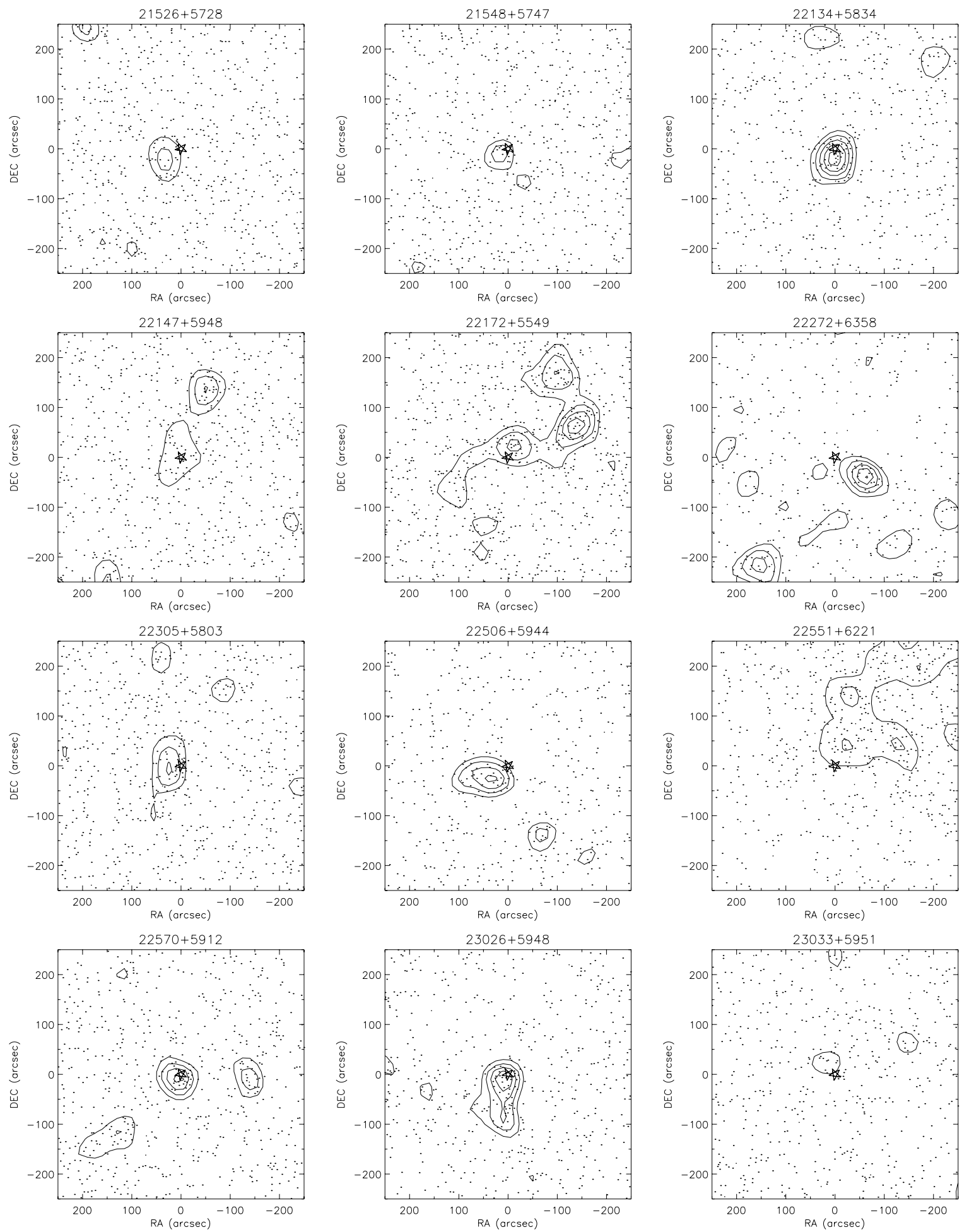

Fig. 1. continued. 
M. S. N. Kumar et al.: The youngest stellar clusters, Online Material p 5
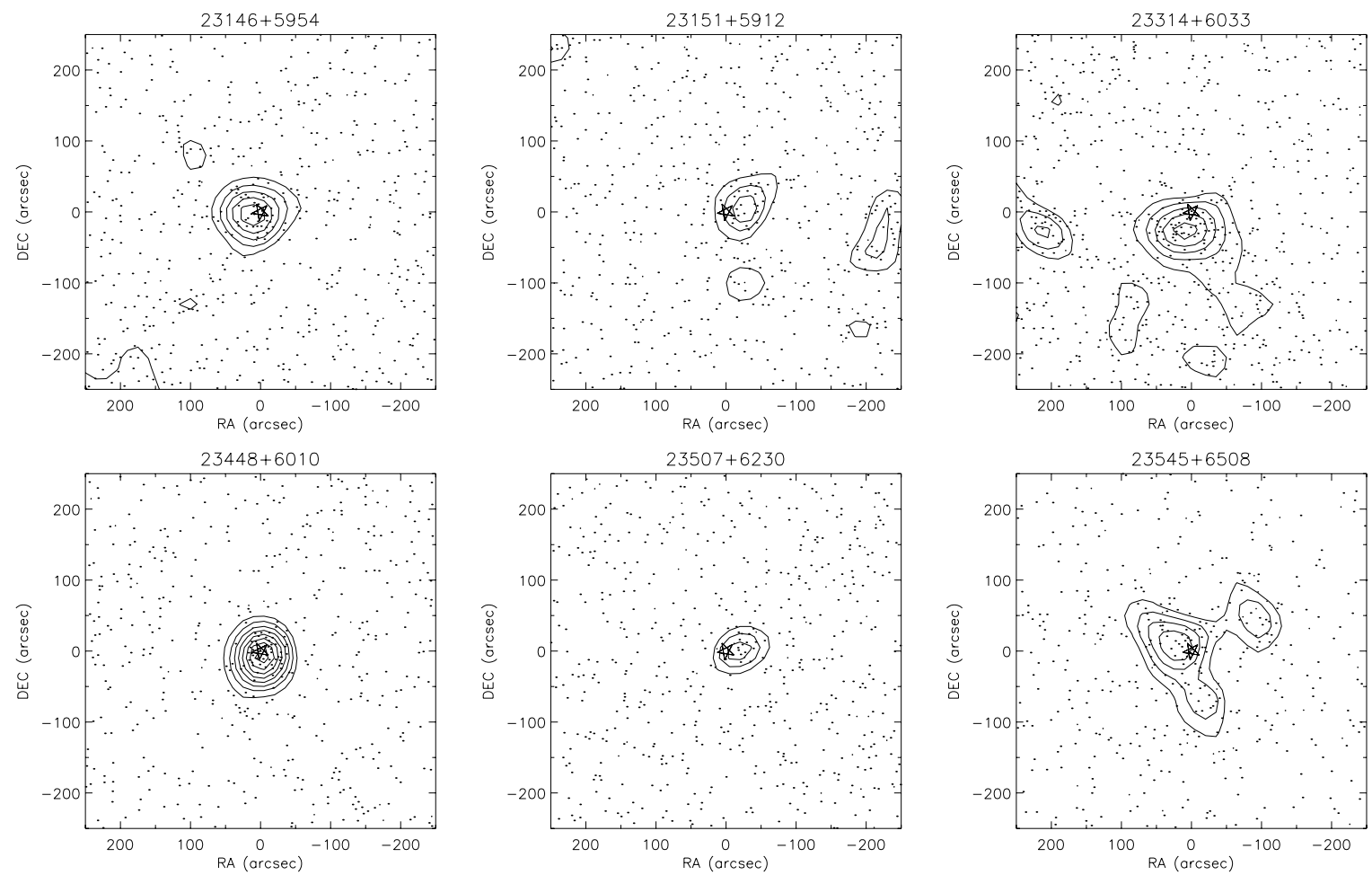

Fig. 1. continued. 